\title{
Synthesis and Co-ordination Chemistry of a New Tripodal Phosphine Alcohol $†$
}

\author{
Tung-Ying Hsieh, Ming-Chu Cheng, Shie-Ming Peng and Shiuh-Tzung Liu• \\ Department of Chemistry, National Taiwan University, Taipei, Taiwan 106, Republic of China
}

\begin{abstract}
The tripodal phosphine alcohol $\mathrm{CH}_{3} \mathrm{C}\left(\mathrm{CH}_{2} \mathrm{PPh}_{2}\right)_{2}\left(\mathrm{CH}_{2} \mathrm{OH}\right)(\mathrm{dpmp})$ was prepared and its co-ordination chemistry towards Group 6 metal carbonyls and a manganese (I) complex studied. In $\left[\mathrm{M}(\mathrm{CO})_{4}-\right.$ $\left.\left(\mathrm{dpmp}-P, P^{\prime}\right)\right](\mathrm{M}=\mathrm{Cr}$, Mo or $\mathrm{W})$, the tripodal ligand behaves as a bidentate ligand with the hydroxyl site unco-ordinated. Reaction of dpmp with $\left[\mathrm{Mn}(\mathrm{CO})_{5} \mathrm{Br}\right]$ gave a stereoisomeric mixture of products, anti,fac- $\left[\mathrm{Mn}(\mathrm{CO})_{3} \mathrm{Br}\left(\mathrm{dpmp}-P, P^{\prime}\right)\right] 4 \mathrm{a}$ and syn,fac- $\left[\mathrm{Mn}(\mathrm{CO})_{3} \mathrm{Br}\left(\mathrm{dpmp}-P, P^{\prime}\right)\right]$ 4b. Treatment of $4 \mathrm{a}$ or $4 \mathrm{~b}$ with silver hexafluorophosphate yielded a facial complex fac- $\left[\mathrm{Mn}(\mathrm{CO})_{3}\left(\mathrm{dpmp}-P, P^{\prime}, O\right)\right] P F_{6} 6$. Complex 6 reacted with the anions $X^{-}\left(X=B r, I, N_{3}\right.$ or $\left.S C N\right)$ stereospecifically to give syn.fac$\left[\mathrm{Mn}(\mathrm{CO})_{3} \mathrm{X}\left(\mathrm{dpmp}-P, P^{\prime}\right)\right]\left(\mathrm{X}=\mathrm{Br} \mathbf{4 b}, \mathbf{1} \mathbf{5 b}, \mathrm{N}_{3} \mathbf{8 b}\right.$ or NCS $\left.9 \mathrm{~b}\right)$. X-Ray crystal structures of complexes $4 \mathrm{~b}$. 6 and $9 \mathrm{~b}$ were determined. The crystal structure of complex $9 \mathrm{~b}$ is the first of a manganese thiocyanate complex containing carbonyl ligands. Both the bond angles $\left[\mathrm{Mn}-\mathrm{N}-\mathrm{C} 170.4(5), \mathrm{N}-\mathrm{C}-\mathrm{S} 175.0(6)^{\circ}\right]$ and the distances [C-N 1.143(8), S-C 1.637(7) A] of 9b clearly demonstrated the nature of the Mn-NCS bond, which is consistent with spectral data. Comparison of the co-ordination chemistry of dpmp with $\mathrm{CH}_{3} \mathrm{C}\left(\mathrm{CH}_{2} \mathrm{PPh}_{2}\right)_{2}\left(\mathrm{CH}_{2} \mathrm{OCH}_{3}\right)$ is discussed.
\end{abstract}

Multidentate ligands containing 'hybrid' donor atoms with consequent modified reactivity of the metal ion have received much attention, especially those containing both soft and hard donors. Among them, ligands with a combination of phosphorus and oxygen donors have been intensively investigated, due to the lability of the ether-metal interaction. ${ }^{1-15}$ We have shown that the tripodal ligand 2,2bis(diphenylphosphinomethyl)propyl methyl ether (dpmpme) behaves in only a bidentate mode towards various metal ions, due to the co-ordinating nature of the ether. ${ }^{14,15}$ In order to investigate the properties of the oxygen donor in this tripodal system the ether site in dpmpme was replaced by a hydroxyl function to alter its capability. Herein we report the results of the co-ordination chemistry of the tripodal ligand 2,2-bis(diphenylphosphinomethyl)propan-1-ol (dpmp) and compare it with that of dpmpme.

\section{Results and Discussion}

The desired tripodal compound dpmp was prepared by the reaction of 3-methyloxetan-3-ylmethyl methanesulfonate with excess of diphenylphosphide anion according to equation (1).

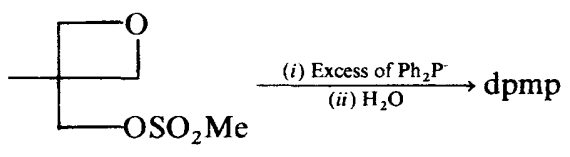

It was isolated as an air-stable white solid and its structure was confirmed by spectral and elemental analyses. A broad absorption at $3378 \mathrm{~cm}^{-1}$ in the IR spectrum clearly indicated the existence of a hydroxyl group and the ${ }^{31} \mathbf{P}$ NMR spectrum showed a single signal at $\delta-25.05$ which is a shift typical of a tertiary phosphine.

Group 6 Complexes.-The thermal reaction of $\left[\mathrm{NEt}_{4}\right]-$ $\left[\mathrm{M}(\mathrm{CO})_{5} \mathrm{Br}\right]$ with dpmp produced $\left[\mathrm{M}(\mathrm{CO})_{4}\left(\mathrm{dpmp}-P, P^{\prime}\right)\right]$

† Supplementary data available: see Instructions for Authors, J. Chem. Soc., Dalton Trans., 1994, Issue 1, pp. xxiii-xxviii.

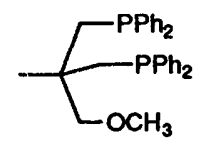

dpmpme

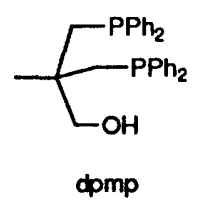

$(\mathrm{M}=\mathrm{Cr}$ 1, Mo 2 or W 3). In all instances, the dpmp tripodal ligand acts in a bidentate manner, essentially similar to that of dpmpme in the analogous complexes $\left[\mathrm{M}(\mathrm{CO})_{4}(\mathrm{dpmpme}-\right.$ $\left.\left.P, P^{\prime}\right)\right](\mathrm{M}=\mathrm{Cr}$, Mo or $\mathrm{W}) \cdot{ }^{14}$ Attempts to prepare the tridentate complexes using $\left[\mathrm{M}(\mathrm{CO})_{3}(\mathrm{MeCN})_{3}\right]$ or $\left[\mathrm{M}(\mathrm{CO})_{3}-\right.$ $\left.\left(\mathrm{C}_{7} \mathrm{H}_{8}\right)\right]$ as starting materials failed. The structural analyses of complexes 1-3, by analogy with $\left[\mathrm{M}(\mathrm{CO})_{4}\left(\mathrm{dpmpme}-P, P^{\prime}\right)\right]$ $(\mathrm{M}=\mathrm{Cr}, \mathrm{Mo}$ or $\mathrm{W}),{ }^{14}$ were confirmed by $\mathrm{IR}$ and NMR spectroscopy (Table 1).

Manganese(I) Complexes.-The substitution reaction of $\left[\mathrm{Mn}(\mathrm{CO})_{5} \mathrm{Br}\right]$ by dpmp in equimolar proportions in refiuxing chloroform yielded a mixture of stereoisomers, anti, fac-[Mn$\left.(\mathrm{CO})_{3} \mathrm{Br}\left(\mathrm{dpmp}-P, P^{\prime}\right)\right] \quad 4 \mathrm{a}$ and $s y n, f a c-\left[\mathrm{Mn}(\mathrm{CO}){ }_{3} \mathrm{Br}(\mathrm{dpmp}-\right.$ $\left.\left.P, P^{\prime}\right)\right] 4 \mathrm{~b}$ in a $1: 1$ ratio [equation (2)]. The isomeric forms of $4 \mathrm{a}$ and $\mathbf{4 b}$ arise from the non-equivalent orientations of the bromide ligand and the unco-ordinating hydroxyl function along the chelate ring. Complex $\mathbf{4 b}$ was obtained in pure form by recrystallization from tetrahydrofuran (thf)-hexane. Both complexes exhibit identical chemical shifts $(\delta 29.7)$ in their ${ }^{31} \mathrm{P}$ NMR spectra and carbonyl absorptions $(2029 \mathrm{~s}, 1963 \mathrm{~m}$ and $1904 \mathrm{~m} \mathrm{~cm}^{-1}$ ) in their IR spectra, but these two complexes are easily differentiated by their ${ }^{1} \mathrm{H}$ NMR spectra. The chemical shift of the methyl group in the syn isomer $4 \mathrm{~b}$ is upfield from that of the anti isomer $4 \mathrm{a}$ by $0.7 \mathrm{ppm}$, whereas the trend of the chemical shifts of the methylene units adjacent to oxygen in $4 \mathrm{a}$ and $4 \mathrm{~b}$ are the opposite ( $c f . \delta 3.304 \mathrm{~b}$ and $2.864 \mathrm{a}$ ). This outcome is consistent with that previously observed for $\left[\mathrm{Mn}(\mathrm{CO})_{3} \mathrm{Br}-\right.$ $\left(\right.$ dpmpsp- $\left.\left.P, P^{\prime}\right)\right][$ dpmpsp $=2,2$-bis (diphenylphosphinomethyl)-1-(phenylsulfanyl)propane]. ${ }^{16}$ The assignment of $4 \mathbf{b}$ as the syn,fac stereoisomer is confirmed by $\mathrm{X}$-ray crystallography (Fig. 1). The analogous iodide complexes $5 \mathbf{a}$ and $\mathbf{5 b}$ were obtained in a similar manner using $\left[\mathrm{Mn}(\mathrm{CO})_{5} \mathrm{I}\right]$ and characterized by spectral methods. 
Table 1 Partial ${ }^{1} \mathrm{H}$ NMR, ${ }^{31} \mathrm{P}$ NMR and IR data of the complexes

\begin{tabular}{|c|c|c|c|c|}
\hline Complex & $\delta\left({ }^{1} \mathrm{H}\right)$ & $\delta\left({ }^{31} \mathrm{P}\right)$ & $\Delta \delta\left({ }^{31} \mathrm{P}\right)^{a}$ & $\tilde{\mathrm{v}}(\mathrm{CO}) / \mathrm{cm}^{-1}$ \\
\hline 1 & $\begin{array}{l}0.71\left(-\mathrm{CH}_{3}\right) \\
3.27\left(-\mathrm{CH}_{2}-\mathrm{O}-\right)\end{array}$ & 40.3 & 65.3 & $\begin{array}{l}2004 \mathrm{~m}, 1947 \mathrm{w} \\
1918 \mathrm{~s}, 1885 \mathrm{~s}\end{array}$ \\
\hline 2 & $\begin{array}{l}0.71\left(-\mathrm{CH}_{3}\right) \\
3.35\left(-\mathrm{CH}_{2}-\mathrm{O}-\right)\end{array}$ & 18.9 & 43.9 & $\begin{array}{l}2017 \mathrm{~m}, 1957 \mathrm{~m} \\
1896 \mathrm{~s}, 1809 \mathrm{~m}\end{array}$ \\
\hline re & $\begin{array}{l}0.73\left(-\mathrm{CH}_{3}\right) \\
3.34\left(-\mathrm{CH}_{2}-\mathrm{O}-\right)\end{array}$ & -0.4 & 24.6 & $\begin{array}{l}2013 \mathrm{~m}, 1948 \mathrm{w} \\
1916 \mathrm{~s}, 1882 \mathrm{~s}\end{array}$ \\
\hline$\left[\mathrm{Cr}(\mathrm{CO})_{4}(\mathrm{dpmpme})\right]^{b}$ & $\begin{array}{l}0.75\left(-\mathrm{CH}_{3}\right) \\
2.76\left(-\mathrm{CH}_{2}-\mathrm{O}-\right)\end{array}$ & 41.1 & 66.2 & $\begin{array}{l}2006 \mathrm{~m}, 1999 \mathrm{~m} \\
1917 \mathrm{~m}, 1882 \mathrm{~s}\end{array}$ \\
\hline$\left[\mathrm{Mo}(\mathrm{CO})_{4}(\mathrm{dpmpme})\right]^{b}$ & $\begin{array}{l}0.75\left(-\mathrm{CH}_{3}\right) \\
2.83\left(-\mathrm{CH}_{2}-\mathrm{O}-\right)\end{array}$ & 19.6 & 40.7 & $\begin{array}{l}2017 \mathrm{~m}, 1919 \mathrm{~m} \\
1892 \mathrm{~s}\end{array}$ \\
\hline$\left[\mathrm{W}(\mathrm{CO})_{4}(\text { dpmpme })\right]^{b}$ & $\begin{array}{l}0.76\left(-\mathrm{CH}_{3}\right) \\
2.82\left(-\mathrm{CH}_{2}-\mathrm{O}-\right)\end{array}$ & 0.01 & 25.1 & $\begin{array}{l}2014 \mathrm{~m}, 1913 \mathrm{~m} \\
1892 \mathrm{~s}\end{array}$ \\
\hline $4 a$ & $\begin{array}{l}1.01\left(-\mathrm{CH}_{3}\right) \\
2.86\left(-\mathrm{CH}_{2}-\mathrm{O}-\right)\end{array}$ & 29.7 & 54.7 & $\begin{array}{l}2029 \mathrm{~s}, 1963 \mathrm{~m} \\
1904 \mathrm{~m}\end{array}$ \\
\hline 4b & $\begin{array}{l}0.26\left(-\mathrm{CH}_{3}\right) \\
3.30\left(-\mathrm{CH}_{2}-\mathrm{O}-\right)\end{array}$ & 29.7 & 54.7 & $\begin{array}{l}2029 \mathrm{~s}, 1963 \mathrm{~m} \\
1904 \mathrm{~m}\end{array}$ \\
\hline $5 \mathbf{a}$ & $\begin{array}{l}1.08\left(-\mathrm{CH}_{3}\right) \\
2.78\left(-\mathrm{CH}_{2}-\mathrm{O}-\right)\end{array}$ & 26.4 & 51.4 & $\begin{array}{l}\text { 2023s, } 1961 \mathrm{~m} \\
1905 \mathrm{~m}\end{array}$ \\
\hline $5 \mathbf{b}$ & $\begin{array}{l}0.16\left(-\mathrm{CH}_{3}\right) \\
3.34\left(-\mathrm{CH}_{2}-\mathrm{O}-\right)\end{array}$ & 26.4 & 51.4 & $\begin{array}{l}2023 \mathrm{~s}, 1961 \mathrm{~m} \\
1905 \mathrm{~m}\end{array}$ \\
\hline 6 & $\begin{array}{l}1.34\left(-\mathrm{CH}_{3}\right) \\
3.71\left(-\mathrm{CH}_{2}-\mathrm{O}-\right)\end{array}$ & 34.8 & 59.8 & $\begin{array}{l}2040 \mathrm{~s}, 1973 \mathrm{~m} \\
1923 \mathrm{~m}\end{array}$ \\
\hline $7 \mathbf{a}$ & $\begin{array}{l}1.12\left(-\mathrm{CH}_{3}\right) \\
3.12\left(-\mathrm{CH}_{2}-\mathrm{O}-\right)\end{array}$ & 30.2 & 54.8 & $\begin{array}{l}2030 \mathrm{~s}, 1966 \mathrm{~m} \\
1907 \mathrm{~m}\end{array}$ \\
\hline $7 \mathrm{~b}$ & $\begin{array}{l}0.46\left(-\mathrm{CH}_{3}\right) \\
3.27\left(-\mathrm{CH}_{2}-\mathrm{O}-\right)\end{array}$ & 29.2 & 53.8 & $\begin{array}{l}2030 \mathrm{~s}, 1966 \mathrm{~m} \\
1907 \mathrm{~m}\end{array}$ \\
\hline $8 b$ & $\begin{array}{l}0.42\left(-\mathrm{CH}_{3}\right) \\
3.23\left(-\mathrm{CH}_{2}-\mathrm{O}-\right)\end{array}$ & 32.7 & 57.7 & $\begin{array}{l}2009 \mathrm{~s}, 1999 \mathrm{~m} \text {, } \\
1948 \mathrm{~m}, 1903 \mathrm{~m}\end{array}$ \\
\hline $9 b$ & $\begin{array}{l}0.45\left(-\mathrm{CH}_{3}\right) \\
3.35\left(-\mathrm{CH}_{2}-\mathrm{O}-\right)\end{array}$ & 33.4 & 58.4 & $\begin{array}{l}2036 \mathrm{~s}, 1968 \mathrm{~m}, \\
1926 \mathrm{~m}\end{array}$ \\
\hline
\end{tabular}

${ }^{a} \Delta \delta=\delta$ (complex $)-\delta($ free ligand $)$ in ppm. ${ }^{b}$ Ref. 14.<smiles>CC(CO)(Cc1ccccc1)Cc1ccccc1</smiles><smiles>CC1(Cc2ccccc2)C[P+]([N+](=O)[O-])P(Br)(Br)(c2ccccc2)C1</smiles>

$\left[\mathrm{Mn}(\mathrm{CO})_{3} \mathrm{Br}\left(\mathrm{dpmpsp}-P, P^{\prime}\right)\right]$

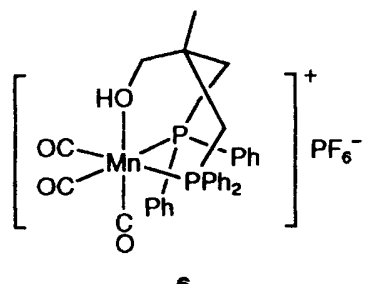

A mixture of $4 \mathbf{a}$ and $4 \mathbf{b}$ was readily converted into the complex fac- $\left[\mathrm{Mn}(\mathrm{CO})_{3}\left(\mathrm{dpmp}-P, P^{\prime}, O\right)\right] \mathrm{PF}_{6} 6$ on treatment with silver hexafluorophosphate, the dpmp ligand now coordinating in a tridentate, rather than bidentate manner. Complex 6 was isolated as an air-stable, yellow crystalline solid and its structure was confirmed by single-crystal X-ray crystallography (Fig. 2). The ${ }^{1} \mathrm{H}$ NMR resonance of the methyl group at $\delta 1.34$ in complex 6 is relatively deshielded compared with that in the other tripodal $\mathrm{CH}_{3} \mathrm{C}\left(\mathrm{CH}_{2} \mathrm{PPh}_{2}\right)_{2}\left(\mathrm{CH}_{2} \mathrm{OR}\right)$ $(\mathrm{R}=\mathrm{H}$ or $\mathrm{Me}$ ) complexes (Table 1$)$, a trend which is consistent with other known systems. ${ }^{18,19}$

The ligand dpmpme behaved similarly to dpmp towards $\left[\mathrm{Mn}(\mathrm{CO})_{5} \mathrm{Br}\right]$ to give a mixture of products $7 \mathrm{a}$ and $7 \mathbf{b}$. Upon treatment with $\mathrm{AgPF}_{6}$, neither $7 \mathbf{a}$ nor $7 \mathbf{b}$ led to the fomation of

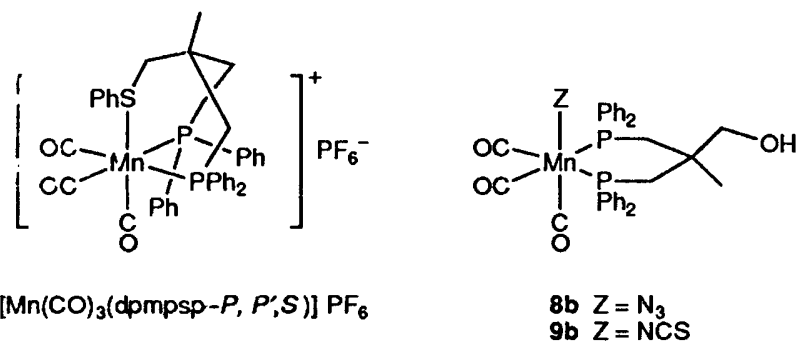

a tridentate-mode complex, indicating that the co-ordinating ability of the ether function is weaker towards the manganese(I) complex than that of the hydroxyl.

Ligand-substitution Reactions of Complex 6.-Reaction of complex 6 with tetrabutylammonium bromide proceeded in thf at room temperature by displacement of the co-ordinated oxygen donor to give syn, $f a c-\left[\mathrm{Mn}(\mathrm{CO})_{3} \mathrm{Br}\left(\mathrm{dpmp}-P, P^{\prime}\right)\right] \mathbf{4 b}$ exclusively. The incoming bromide evidently co-ordinates at the position that the oxygen donor occupied. Stereospecificity of this kind in a ligand-substitution reaction is relevant to the previously reported system, $\left[\mathrm{Mn}(\mathrm{CO})_{3}\left(\mathrm{dpmpsp}-P, P^{\prime}, S\right)\right] \mathrm{PF}_{6}$. Moreover, complex 6 reacted with iodide, azide or thiocyanate 


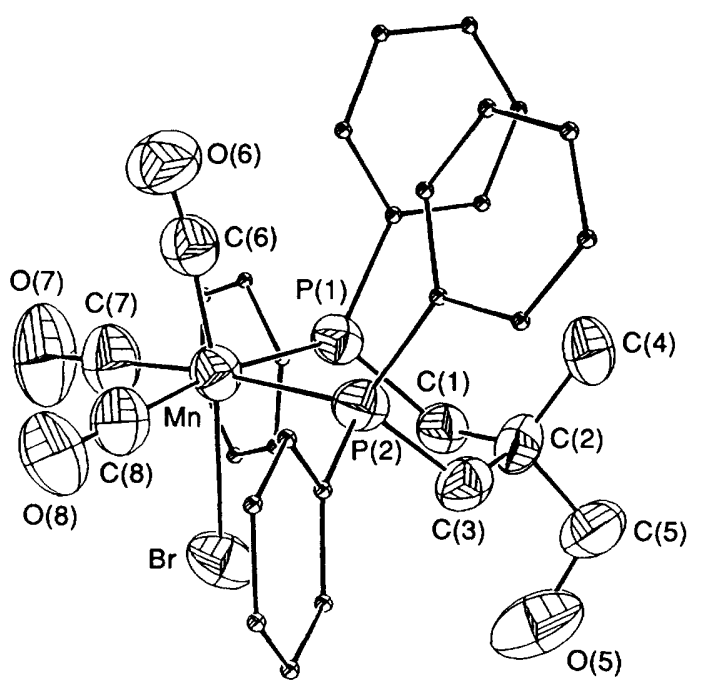

Fig. 1 ORTEP ${ }^{17}$ plot of $s y n, f a c-\left[\mathrm{Mn}(\mathrm{CO}){ }_{3} \operatorname{Br}\left(\mathrm{dpmp}-P, P^{\prime}\right)\right] \mathbf{4 b}$

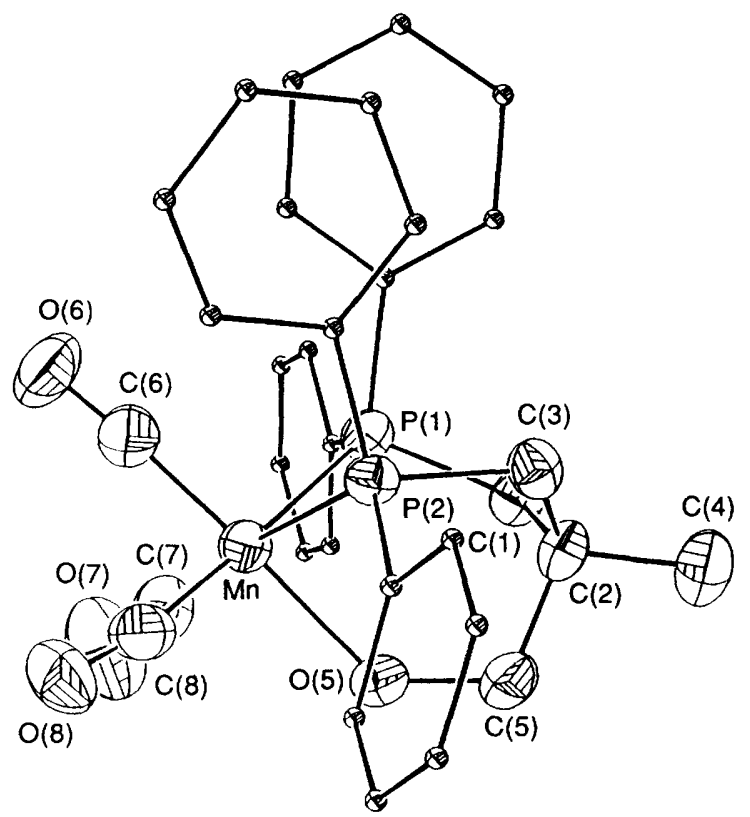

Fig. 2 ORTEP ${ }^{17}$ plot of the $\left[\mathrm{Mn}(\mathrm{CO})_{3}\left(\mathrm{dpmp}-P, P^{\prime}, O\right)\right]^{+}$cation of 6

stereospecifically to give the corresponding syn products $\mathbf{5 b}$, $\left[\mathrm{Mn}(\mathrm{CO})_{3}\left(\mathrm{~N}_{3}\right)\left(\mathrm{dpmp}-P, P^{\prime}\right)\right] \mathbf{8 b}$ or $\left[\mathrm{Mn}(\mathrm{CO})_{3}(\mathrm{NCS})(\mathrm{dpmp}-\right.$ $\left.\left.P, P^{\prime}\right)\right] 9$ b respectively. Both complexes $8 \mathrm{~b}$ and $9 \mathrm{~b}$ were characterized by spectral and elemental analyses and the stereochemistry of $\mathbf{9 b}$ was confirmed by X-ray structural analysis (Fig. 3).

Crystal Structure Analysis.-The structures of complexes $\mathbf{4 b}$, 6 and $9 b$ were determined by single-crystal X-ray diffraction and are shown in Figs. 1-3. Selected bond distances and angles are listed in Table 2 and atomic coordinates in Tables 3-5, respectively. These structural determinations demonstrate that each complex consists of an octahedrally co-ordinated manganese atom with three carbonyl ligands arranged in a facial fashion. In complex 6, all three donors (two phosphorus and one oxygen atoms) of the tripodal ligand form another facial plane around the metal centre. The metal to phosphorus distances are all within normal ranges [2.329(2)-2.371(2) \&]. As expected for the trans influence of ligands, the $\mathrm{M}-\mathrm{C}$ distances trans to the phosphines are slightly larger than those trans to the bromine, oxygen or nitrogen donor. The deviation of $\mathrm{P}(1)-\mathrm{Mn}-\mathrm{C}(6)$ from $90^{\circ}$ in both $\mathbf{4 b}$ and $9 \mathrm{~b}$ is due to steric interaction of the phenyl ring in the phosphorus and carbonyl ligand $[\mathrm{C}(6)-\mathrm{O}(6)]$.
Table 2 Selected bond distances $(\AA)$ and angles $\left({ }^{\circ}\right)$

\begin{tabular}{lccc} 
& $\mathbf{4 b}(\mathrm{X}=\mathrm{Br})$ & $\mathbf{6}[\mathrm{X}=\mathrm{O}(5)]$ & $\mathbf{9 b}(\mathrm{X}=\mathbf{N})$ \\
$\mathrm{Mn}-\mathrm{P}(1)$ & $2.340(2)$ & $2.348(2)$ & $2.359(2)$ \\
$\mathrm{Mn}-\mathrm{P}(2)$ & $2.371(2)$ & $2.329(2)$ & $2.346(3)$ \\
$\mathrm{Mn}-\mathrm{X}$ & $2.543(1)$ & $2.102(3)$ & $2.015(5)$ \\
$\mathrm{Mn}-\mathrm{C}(6)$ & $1.762(7)$ & $1.767(5)$ & $1.783(7)$ \\
$\mathrm{Mn}-\mathrm{C}(7)$ & $1.816(7)$ & $1.812(5)$ & $1.810(7)$ \\
$\mathrm{Mn}-\mathrm{C}(8)$ & $1.809(7)$ & $1.813(5)$ & $1.823(7)$ \\
$\mathrm{C}(6)-\mathrm{O}(6)$ & $1.152(8)$ & $1.145(6)$ & $1.157(8)$ \\
$\mathrm{C}(7)-\mathrm{O}(7)$ & $1.131(8)$ & $1.136(6)$ & $1.152(8)$ \\
$\mathrm{C}(8)-\mathrm{O}(8)$ & $1.142(8)$ & $1.156(6)$ & $1.143(8)$ \\
S-C & - & - & $1.637(7)$ \\
$\mathrm{C}-\mathrm{N}$ & - & - & $1.143(8)$ \\
& & & \\
$\mathrm{P}(1)-\mathrm{Mn}-\mathrm{P}(2)$ & $88.2(2)$ & $87.03(6)$ & $87.70(8)$ \\
$\mathrm{P}(1)-\mathrm{Mn}-\mathrm{X}$ & $87.87(6)$ & $85.0(1)$ & $86.1(2)$ \\
$\mathrm{P}(2)-\mathrm{Mn}-\mathrm{X}$ & $88.33(6)$ & $82.8(1)$ & $84.0(2)$ \\
$\mathrm{P}(1)-\mathrm{Mn}-\mathrm{C}(6)$ & $100.9(2)$ & $94.2(2)$ & $98.5(2)$ \\
$\mathrm{P}(1)-\mathrm{Mn}-\mathrm{C}(7)$ & $90.7(2)$ & $92.4(2)$ & $90.0(2)$ \\
$\mathrm{P}(1)-\mathrm{Mn}-\mathrm{C}(8)$ & $171.0(2)$ & $177.6(2)$ & $174.7(2)$ \\
$\mathrm{P}(2)-\mathrm{Mn}-\mathrm{C}(6)$ & $95.8(2)$ & $98.8(2)$ & $93.3(2)$ \\
$\mathrm{P}(2)-\mathrm{Mn}-\mathrm{C}(7)$ & $174.3(2)$ & $173.2(2)$ & $176.4(2)$ \\
$\mathrm{P}(2)-\mathrm{Mn}-\mathrm{C}(8)$ & $91.0(2)$ & $91.8(2)$ & $92.7(2)$ \\
C(6)-Mn-C(7) & $89.9(3)$ & $88.0(2)$ & $89.8(3)$ \\
C(6)-Mn-C(8) & $88.1(3)$ & $84.0(2)$ & $86.7(3)$ \\
C(7)-Mn-C(8) & $89.3(3)$ & $89.1(2)$ & $89.4(3)$ \\
$\mathrm{Mn}-\mathrm{N}-\mathrm{C}$ & - & - & $170.4(5)$ \\
$\mathrm{N}-\mathrm{C}-\mathrm{S}$ & - & - & $175.0(6)$ \\
\hline
\end{tabular}

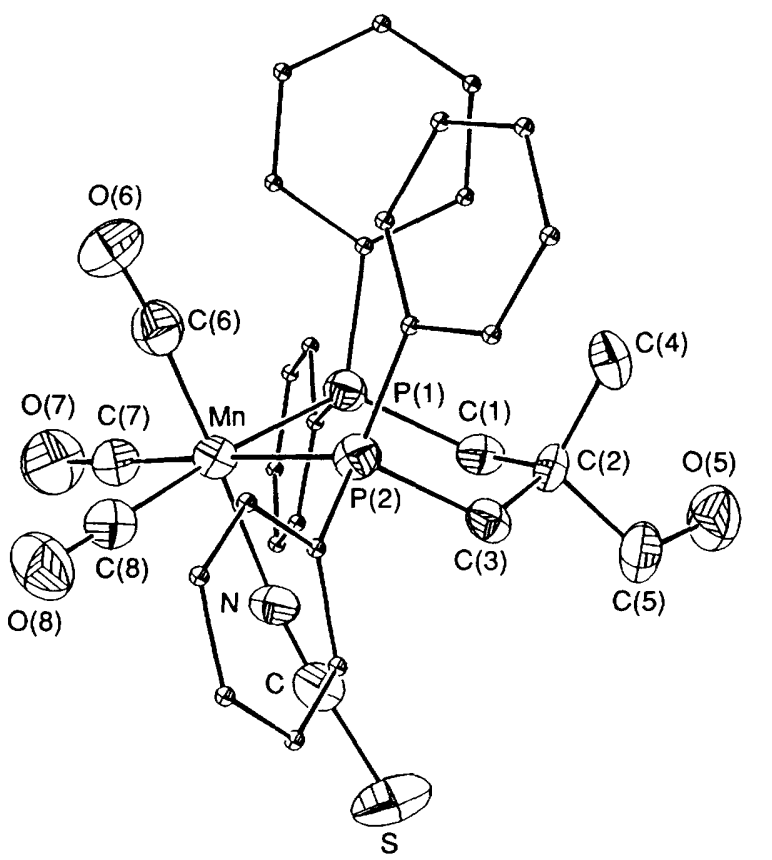

Fig. 3 ORTEP ${ }^{17}$ plot of $s y n, f a c-\left[\mathrm{Mn}(\mathrm{CO})_{3}(\mathrm{NCS})\left(\mathrm{dpmp}-P, P^{\prime}\right)\right]$ 9b

The crystal structure of $\mathbf{9 b}$ is the first of a manganese thiocyanate compound containing $\pi$-acceptor ligands. The $\mathrm{Mn}-\mathrm{N}-\mathrm{C}\left[170.4(5)^{\circ}\right]$ and $\mathrm{N}-\mathrm{C}-\mathrm{S}\left[175.0(6)^{\circ}\right]$ angles imply that the co-ordinating thiocyanate ligand is linear, similar to that in trans- $\left[\mathrm{Mn}\left(\mathrm{H}_{2} \mathrm{O}\right)_{4}(\mathrm{NCS})_{2}\right]\left(171.9\right.$ and $178.5^{\circ}$ respectively). ${ }^{20}$ The $\mathrm{C}-\mathrm{N}[1.143(8) \AA]$ and $\mathrm{S}-\mathrm{C}[1.637(7) \AA]$ bond distances, which are essentially identical to those of trans-[Mn$\left.\left(\mathrm{H}_{2} \mathrm{O}\right)_{4}(\mathrm{NCS})_{2}\right](\mathrm{N}-\mathrm{C} 1.146, \mathrm{~S}-\mathrm{C} 1.637 \AA)$, indicate triple and single bonds respectively. This observation is clearly considered indicative of Mn-NCS bonding, which is consistent with the IR absorption at $2105 \mathrm{~cm}^{-1}$ for the $\mathrm{CN}$ of the thiocyanato- $N$ complex 9b. ${ }^{21,22}$ The bands at 2036, 1968 and $1926 \mathrm{~cm}^{-1}$ (carbonyl stretches) support the structure of $9 \mathrm{~b}$ in which all the carbonyl ligands are cis. 
Table 3 Atomic coordinates for complex $4 \mathbf{b}$

\begin{tabular}{|c|c|c|c|}
\hline Atom & $x$ & $y$ & $z$ \\
\hline $\mathrm{Mn}$ & $0.76913(9)$ & $0.51281(6)$ & $0.27702(5)$ \\
\hline $\mathrm{Br}$ & $0.57634(7)$ & $0.58028(5)$ & $0.21369(4)$ \\
\hline $\mathbf{P}(1)$ & $0.67606(15)$ & $0.39272(10)$ & $0.23507(9)$ \\
\hline $\mathrm{P}(2)$ & $0.69208(16)$ & $0.50074(10)$ & $0.38536(9)$ \\
\hline$C(1)$ & $0.5244(5)$ & $0.3838(4)$ & $0.2514(3)$ \\
\hline$C(2)$ & $0.4993(5)$ & $0.3897(4)$ & $0.3311(3)$ \\
\hline$C(3)$ & $0.5374(5)$ & $0.4691(4)$ & $0.3678(3)$ \\
\hline$C(4)$ & $0.5489(6)$ & $0.3222(4)$ & $0.3809(3)$ \\
\hline$C(5)$ & $0.3625(6)$ & $0.3839(5)$ & $0.3212(4)$ \\
\hline$O(5)$ & $0.3080(4)$ & $0.4531(4)$ & $0.2832(3)$ \\
\hline$C(6)$ & $0.9142(6)$ & $0.4811(4)$ & $0.3190(4)$ \\
\hline $\mathrm{O}(6)$ & $1.0125(4)$ & $0.4660(3)$ & $0.3441(3)$ \\
\hline$C(7)$ & $0.8142(7)$ & $0.5254(4)$ & $0.1881(4)$ \\
\hline $\mathrm{O}(7)$ & $0.8451(6)$ & $0.5360(3)$ & $0.1342(3)$ \\
\hline$C(8)$ & $0.8177(6)$ & $0.6113(4)$ & $0.3026(4)$ \\
\hline $\mathrm{O}(8)$ & $0.8489(5)$ & $0.6739(3)$ & $0.3157(3)$ \\
\hline$C(11 A)$ & $0.6480(5)$ & $0.3816(4)$ & $0.1321(3)$ \\
\hline$C(12 A)$ & $0.5502(6)$ & $0.4125(4)$ & $0.0854(3)$ \\
\hline$C(13 A)$ & $0.5327(6)$ & $0.4047(4)$ & $0.0079(3)$ \\
\hline$C(14 A)$ & $0.6127(7)$ & $0.3663(4)$ & $-0.0225(3)$ \\
\hline$C(15 A)$ & $0.7125(7)$ & $0.3354(5)$ & $0.0226(4)$ \\
\hline$C(16 A)$ & $0.7298(6)$ & $0.3417(4)$ & $0.1008(3)$ \\
\hline$C(11 B)$ & $0.7509(5)$ & $0.2998(4)$ & $0.2643(3)$ \\
\hline$C(12 B)$ & $0.6868(6)$ & $0.2306(4)$ & $0.2630(4)$ \\
\hline$C(13 B)$ & $0.7451(7)$ & $0.1608(4)$ & $0.2822(4)$ \\
\hline$C(14 B)$ & $0.8672(7)$ & $0.1587(4)$ & $0.3023(4)$ \\
\hline$C(15 B)$ & $0.9326(6)$ & $0.2257(4)$ & $0.3027(4)$ \\
\hline$C(16 B)$ & $0.8732(6)$ & $0.2953(4)$ & $0.2839(4)$ \\
\hline$C(21 A)$ & $0.6852(6)$ & $0.5948(4)$ & $0.4319(3)$ \\
\hline$C(22 A)$ & $0.5868(6)$ & $0.6437(4)$ & $0.4123(4)$ \\
\hline$C(23 A)$ & $0.5869(7)$ & $0.7149(4)$ & $0.4488(4)$ \\
\hline$C(24 A)$ & $0.6814(7)$ & $0.7369(4)$ & $0.5028(4)$ \\
\hline$C(25 \mathrm{~A})$ & $0.7800(7)$ & $0.6900(4)$ & $0.5224(4)$ \\
\hline$C(26 A)$ & $0.7826(6)$ & $0.6189(4)$ & $0.4874(4)$ \\
\hline$C(2 \mid B)$ & $0.7638(5)$ & $0.4409(4)$ & $0.4655(3)$ \\
\hline$C(22 B)$ & $0.7226(6)$ & $0.4449(4)$ & $0.5319(4)$ \\
\hline$C(23 B)$ & $0.7715(6)$ & $0.4004(4)$ & $0.5929(3)$ \\
\hline$C(24 B)$ & $0.8625(6)$ & $0.3513(4)$ & $0.5897(4)$ \\
\hline $\mathrm{C}(25 \mathrm{~B})$ & $0.9040(6)$ & $0.3456(4)$ & $0.5244(4)$ \\
\hline$C(26 B)$ & $0.8547(6)$ & $0.3900(4)$ & $0.4628(3)$ \\
\hline$O(31)$ & $0.2128(9)$ & $0.4462(8)$ & $0.0816(6)$ \\
\hline$C(32)$ & $0.1869(14)$ & $0.4339(9)$ & $0.1416(6)$ \\
\hline$C(33)$ & $0.0903(13)$ & $0.3994(10)$ & $0.1466(7)$ \\
\hline$C(34)$ & $0.0961(16)$ & $0.3571(7)$ & $0.0900(9)$ \\
\hline$C(35)$ & $0.1016(14)$ & $0.4120(11)$ & $0.0393(7)$ \\
\hline
\end{tabular}

Table 6 lists all the torsional angles around the six-membered chelate ring of the complexes, which facilitates their comparison. The conformations of the chelate rings in $\mathbf{4 b}$ and $9 \mathrm{~b}$ adopt the chair forms according to the characteristic $+g,-g$ alternations of the torsional angles along the chelate rings. In contrast, all three 'fused' rings in $\mathbf{6}$ are forced into twist-boat forms because of the geometric limitation of the tripodal frame.

\section{Conclusion}

In this work we investigated the co-ordination behaviour of tripodal ether and hydroxyl phosphines towards some transition-metal ions. Even through a chelation effect, the oxygen atoms in both tripodal ligands (dpmp and dpmpme) are unable to co-ordinate to metal ions of Group 6 carbonyls, but the hydroxyl function of dpmp becomes a donor towards $\mathrm{Mn}^{1}$ in the formation of $f a c-\left[\mathrm{Mn}(\mathrm{CO})_{3}\left(\mathrm{dpmp}-P, P^{\prime}, O\right)\right] \mathrm{PF}_{6}$. This complex undergoes ligand-substitution reactions with halide or pseudo-halide ligands stereospecifically to provide syn, fac$\left[\mathrm{Mn}(\mathrm{CO})_{3} \mathrm{X}\left(\mathrm{dpmp}-P, P^{\prime}\right)\right]\left(\mathrm{X}=\mathrm{Br}, \mathrm{I}, \mathrm{NCS}\right.$ or $\left.\mathrm{N}_{3}\right)$. The first $\mathrm{X}$-ray crystal structure determination of a manganese(I) thiocyanate compound containing carbonyl ligands, syn, fac$\left[\mathrm{Mn}(\mathrm{CO})_{3}(\mathrm{NCS})\left(\mathrm{dpmp}-P, P^{\prime}\right)\right]$, accommodates accurately the $\mathrm{Mn}-\mathrm{NCS}$ bonding mode.
Table 4 Atomic coordinates for complex 6

\begin{tabular}{|c|c|c|c|}
\hline Atom & $x$ & $y$ & $z$ \\
\hline $\mathrm{Mn}$ & 0.51264 & $0.46536(7)$ & 0.37230 \\
\hline $\mathrm{P}(1)$ & $0.46780(7)$ & $0.37227(13)$ & $0.45971(8)$ \\
\hline $\mathrm{P}(2)$ & $0.60081(7)$ & $0.54869(14)$ & $0.48034(8)$ \\
\hline$C(1)$ & $0.44601(23)$ & $0.5122(5)$ & $0.5101(3)$ \\
\hline$C(2)$ & $0.49202(25)$ & $0.6358(5)$ & $0.5258(3)$ \\
\hline$C(3)$ & $0.56809(24)$ & $0.5989(5)$ & $0.5553(3)$ \\
\hline$C(4)$ & $0.4798(3)$ & $0.7244(6)$ & $0.5882(3)$ \\
\hline$C(5)$ & $0.4701(3)$ & $0.7218(5)$ & $0.4516(3)$ \\
\hline$O(5)$ & $0.46404(16)$ & $0.6431(3)$ & $0.38512(18)$ \\
\hline$C(6)$ & $0.5509(3)$ & $0.3135(5)$ & $0.3601(3)$ \\
\hline$O(6)$ & $0.57416(21)$ & $0.2172(4)$ & $0.34664(24)$ \\
\hline$C(7)$ & $0.4385(3)$ & $0.4184(6)$ & $0.2889(3)$ \\
\hline$O(7)$ & $0.39561(21)$ & $0.3878(5)$ & $0.23331(23)$ \\
\hline$C(8)$ & $0.55044(25)$ & $0.5332(5)$ & $0.3068(3)$ \\
\hline $\mathrm{O}(8)$ & $0.57475(21)$ & $0.5569(4)$ & $0.26164(21)$ \\
\hline$C(11 A)$ & $0.52041(23)$ & $0.2572(5)$ & $0.5333(3)$ \\
\hline$C(12 A)$ & $0.555 \mathrm{l}(3)$ & $0.1558(6)$ & $0.5140(3)$ \\
\hline$C(13 A)$ & $0.5936(3)$ & $0.0621(6)$ & $0.5672(4)$ \\
\hline$C(14 A)$ & $0.5986(3)$ & $0.0701(6)$ & $0.6423(3)$ \\
\hline$C(15 A)$ & $0.5633(3)$ & $0.1702(6)$ & $0.6634(3)$ \\
\hline$C(16 A)$ & $0.5255(3)$ & $0.2619(6)$ & $0.6097(3)$ \\
\hline$C(11 B)$ & $0.38764(23)$ & $0.2836(5)$ & $0.4182(3)$ \\
\hline$C(12 B)$ & $0.3794(3)$ & $0.1506(5)$ & $0.4357(3)$ \\
\hline$C(13 B)$ & $0.3170(3)$ & $0.0883(6)$ & $0.4001(4)$ \\
\hline$C(14 B)$ & $0.2635(3)$ & $0.1568(6)$ & $0.3503(3)$ \\
\hline$C(15 B)$ & $0.2707(3)$ & $0.2866(6)$ & $0.3343(3)$ \\
\hline$C(16 B)$ & $0.3322(3)$ & $0.3512(5)$ & $0.3674(3)$ \\
\hline$C(21 A)$ & $0.67188(23)$ & $0.4360(5)$ & $0.5260(3)$ \\
\hline$C(22 A)$ & $0.70510(23)$ & $0.3886(5)$ & $0.4794(3)$ \\
\hline$C(23 A)$ & $0.7586(3)$ & $0.3006(6)$ & $0.5064(3)$ \\
\hline$C(24 \mathrm{~A})$ & $0.7799(3)$ & $0.2575(6)$ & $0.5815(3)$ \\
\hline$C(25 A)$ & $0.7482(3)$ & $0.3043(6)$ & $0.6297(3)$ \\
\hline$C(26 A)$ & $0.6949(3)$ & $0.3970(6)$ & $0.6025(3)$ \\
\hline$C(21 B)$ & $0.64142(23)$ & $0.7053(5)$ & $0.4673(3)$ \\
\hline$C(22 B)$ & $0.7011(3)$ & $0.7440(6)$ & $0.5237(3)$ \\
\hline$C(23 B)$ & $0.7320(3)$ & $0.8647(6)$ & $0.5178(3)$ \\
\hline$C(24 B)$ & $0.7020(3)$ & $0.9450(6)$ & $0.4537(3)$ \\
\hline$C(25 B)$ & $0.6419(3)$ & $0.9068(5)$ & $0.3971(3)$ \\
\hline $\mathrm{C}(26 \mathrm{~B})$ & $0.61102(25)$ & $0.7896(5)$ & $0.4023(3)$ \\
\hline $\mathrm{P}$ & $0.24341(9)$ & $0.73085(17)$ & $0.26866(10)$ \\
\hline$F(1)$ & $0.26939(24)$ & $0.8473(4)$ & $0.2293(3)$ \\
\hline$F(2)$ & $0.31055(18)$ & $0.7388(6)$ & $0.34098(23)$ \\
\hline$F(3)$ & $0.2767(3)$ & $0.6256(4)$ & $0.2331(3)$ \\
\hline$F(4)$ & $0.21654(21)$ & $0.6159(4)$ & $0.30591(24)$ \\
\hline$F(5)$ & $0.17762(19)$ & $0.7245(4)$ & $0.19581(23)$ \\
\hline$F(6)$ & $0.21179(21)$ & $0.8386(4)$ & $0.3067(3)$ \\
\hline$C(30)$ & $0.4142(3)$ & $0.8777(6)$ & $0.2252(3)$ \\
\hline $\mathrm{O}(31)$ & $0.43519(20)$ & $0.7687(4)$ & $0.25024(23)$ \\
\hline$C(32)$ & $0.4193(4)$ & $0.9909(8)$ & $0.2765(5)$ \\
\hline$C(33)$ & $0.3809(3)$ & $0.8967(8)$ & $0.1416(4)$ \\
\hline
\end{tabular}

\section{Experimental}

Proton ${ }^{13} \mathrm{C}$ and ${ }^{31} \mathrm{P}$ NMR spectra were measured on a Bruker AC-E 200 or AM-300WB spectrometer. Chemical shifts are given in ppm relative to $\mathrm{SiMe}_{4}$ and $85 \% \mathrm{H}_{3} \mathrm{PO}_{4}$ for ${ }^{1} \mathrm{H}$ and ${ }^{31} \mathrm{P}$ NMR spectra in $\mathrm{CDCl}_{3}$ respectively, unless otherwise noted. Infrared spectra were obtained on Perkin-Elmer $983 \mathrm{G}$ and BioRad FT-IR instruments. Elemental analyses were performed on a Perkin-Elmer $240 \mathrm{C}$ instrument.

Syntheses.-2,2-Bis(diphenylphosphinomethyl) propan-1-ol $(d p m p)$. Diphenylphosphide anion, which was prepared by the addition of a hexane solution $\left(1.6 \mathrm{~mol} \mathrm{dm}^{-3}\right)$ of $n$-butyllithium $\left(27 \mathrm{~cm}^{3}, 43.2 \mathrm{mmol}\right)$ to a thf solution of diphenylphosphine $(5.5 \mathrm{~g}, 29 \mathrm{mmol})$ at ice temperature, was added to a thf $\left(50 \mathrm{~cm}^{3}\right)$ solution of 3-methyloxetan-3-ylmethyl methanesulfonate ${ }^{9}(2.5$ $\mathrm{g}, 13.9 \mathrm{mmol}$ ) with stirring. The resulting mixture was heated to reflux for $6 \mathrm{~h}$ and degassed water $\left(10 \mathrm{~cm}^{3}\right)$ was added to quench the reaction. The organic portion was separated, dried and concentrated. The residue was chromatographed on silica $(80 \mathrm{~g})$ 
with ethyl acetate-hexane $(1: 3)$ as eluent. The eluate was collected and concentrated to give the desired product as a white solid ( $3.34 \mathrm{~g}, 53 \%$ ) (Found: C, 76.10; H, 6.70. Calc. for $\mathrm{C}_{29} \mathrm{H}_{30} \mathrm{OP}_{2}$ : C, $76.30 ; \mathrm{H}, 6.65 \%$ ), m.p. $96-98^{\circ} \mathrm{C}$. IR (KBr): $v(\mathrm{OH}) 3378 \mathrm{~cm}^{-1}$. NMR: ${ }^{1} \mathrm{H}, \delta 7.57-7.36(\mathrm{~m}, 20 \mathrm{H}), 3.60(\mathrm{~s}, 2 \mathrm{H})$, $2.52(\mathrm{t}, 4 \mathrm{H})$ and $1.09(\mathrm{~s}, 3 \mathrm{H}) ;{ }^{31} \mathrm{P}, \delta-25.05$.

Table 5 Atomic coordinates for complex 9b

\begin{tabular}{|c|c|c|c|}
\hline Atom & $x$ & $y$ & $z$ \\
\hline $\mathrm{Mn}$ & $0.14134(11)$ & $0.69003(9)$ & $0.32548(8)$ \\
\hline$P(1)$ & $0.19794(18)$ & $0.86647(16)$ & $0.26686(13)$ \\
\hline $\mathrm{P}(2)$ & $0.33481(19)$ & $0.59892(16)$ & $0.20861(13)$ \\
\hline $\mathrm{S}$ & $-0.0813(3)$ & $0.78972(22)$ & $0.03996(17)$ \\
\hline $\mathrm{C}$ & $-0.0247(7)$ & $0.7599(6)$ & $0.1454(5)$ \\
\hline$N$ & $0.0241(5)$ & $0.7404(5)$ & $0.2153(4)$ \\
\hline$C(1)$ & $0.2394(7)$ & $0.8990(6)$ & $0.1327(5)$ \\
\hline$C(2)$ & $0.3522(7)$ & $0.8087(6)$ & $0.0674(4)$ \\
\hline$C(3)$ & $0.3330(6)$ & $0.6860(6)$ & $0.0863(5)$ \\
\hline$C(4)$ & $0.5110(7)$ & $0.7954(6)$ & $0.0701(5)$ \\
\hline$C(5)$ & $0.3228(8)$ & $0.8589(6)$ & $-0.0384(5)$ \\
\hline $\mathrm{O}(5)$ & $0.3489(5)$ & $0.9671(5)$ & $-0.0773(4)$ \\
\hline$C(6)$ & $0.2483(7)$ & $0.6312(6)$ & $0.4218(5)$ \\
\hline$O(6)$ & $0.3093(6)$ & $0.5874(5)$ & $0.4882(4)$ \\
\hline$C(7)$ & $-0.0121(7)$ & $0.7680(6)$ & $0.4095(4)$ \\
\hline$O(7)$ & $-0.1069(5)$ & $0.8143(5)$ & $0.4663(4)$ \\
\hline$C(8)$ & $0.0852(7)$ & $0.5589(6)$ & $0.3632(5)$ \\
\hline$O(8)$ & $0.0486(6)$ & $0.4775(5)$ & $0.3886(4)$ \\
\hline$C(11 \mathrm{~A})$ & $0.0442(6)$ & $1.0030(6)$ & $0.2879(5)$ \\
\hline$C(12 A)$ & $0.0535(7)$ & $1.0879(6)$ & $0.3350(5)$ \\
\hline$C(13 A)$ & $-0.0647(8)$ & $1.1917(6)$ & $0.3485(6)$ \\
\hline$C(14 A)$ & $-0.1907(7)$ & $1.2112(6)$ & $0.3156(5)$ \\
\hline$C(15 A)$ & $-0.2018(7)$ & $1.1271(7)$ & $0.2704(6)$ \\
\hline$C(16 A)$ & $-0.0856(7)$ & $1.0241(6)$ & $0.2562(5)$ \\
\hline$C(11 B)$ & $0.3376(6)$ & $0.8828(5)$ & $0.3199(5)$ \\
\hline$C(12 B)$ & $0.3305(7)$ & $0.8568(7)$ & $0.4212(5)$ \\
\hline$C(13 B)$ & $0.4356(8)$ & $0.8647(7)$ & $0.4649(6)$ \\
\hline$C(14 B)$ & $0.5495(8)$ & $0.8982(7)$ & $0.4098(6)$ \\
\hline$C(15 B)$ & $0.5563(7)$ & $0.9268(7)$ & $0.3121(6)$ \\
\hline$C(16 B)$ & $0.4493(7)$ & $0.9225(6)$ & $0.2656(5)$ \\
\hline$C(21 A)$ & $0.5275(6)$ & $0.5511(6)$ & $0.2220(5)$ \\
\hline$C(22 A)$ & $0.5717(7)$ & $0.5897(6)$ & $0.2898(5)$ \\
\hline$C(23 A)$ & $0.7193(7)$ & $0.5662(7)$ & $0.2887(6)$ \\
\hline$C(24 A)$ & $0.8210(7)$ & $0.5054(7)$ & $0.2195(6)$ \\
\hline$C(25 A)$ & $0.7769(7)$ & $0.4636(7)$ & $0.1529(6)$ \\
\hline$C(26 A)$ & $0.6318(7)$ & $0.4858(6)$ & $0.1539(5)$ \\
\hline$C(21 B)$ & $0.3165(7)$ & $0.4635(6)$ & $0.190 !(5)$ \\
\hline$C(22 B)$ & $0.3724(8)$ & $0.3585(6)$ & $0.2524(6)$ \\
\hline $\mathrm{C}(23 \mathrm{~B})$ & $0.3465(9)$ & $0.2558(7)$ & $0.2476(7)$ \\
\hline$C(24 B)$ & $0.2659(9)$ & $0.2584(7)$ & $0.1806(7)$ \\
\hline$C(25 B)$ & $0.2109(8)$ & $0.3601(7)$ & $0.1193(6)$ \\
\hline$C(26 B)$ & $0.2339(7)$ & $0.4627(6)$ & $0.1236(5)$ \\
\hline
\end{tabular}

$\left[\mathrm{M}(\mathrm{CO})_{4}\left(\mathrm{dpmp}-P, P^{\prime}\right)\right](\mathrm{M}=\mathrm{Cr} \mathbf{1}$, Mo 2 or $\mathrm{W} 3)$. The carbonyl complex $\left[\mathrm{NEt}_{4}\right]\left[\mathrm{M}(\mathrm{CO})_{5} \mathrm{Br}\right]$ and dpmp in equimolar proportions in thf were heated to reflux for $3 \mathrm{~h}$. Filtration and concentration of the reaction mixture gave the crude product as a brown viscous liquid. The pure product was obtained by chromatography on silica gel with acetone as the eluent.

[2,2-Bis(diphenylphosphinomethyl)propan-1-ol- $\left.P, P^{\prime}\right]$ tetracarbonylchromium $(0)$ 1. Yellow solid (96\%) (Found: C, 63.65; H, 4.95. Calc. for $\mathrm{C}_{33} \mathrm{H}_{30} \mathrm{CrO}_{5} \mathrm{P}_{2}$ : C, 63.85; H, 4.85\%), m.p. 160-164 ${ }^{\circ} \mathrm{C}$ (decomp.). IR (benzene): $v_{\mathrm{CO}} 2004,1947,1918$ and $1885 \mathrm{~cm}^{-1}$. NMR: ${ }^{1} \mathrm{H}, \delta 7.82-7.75(\mathrm{~m}, 4 \mathrm{H}), 7.54-7.34(\mathrm{~m}, 16 \mathrm{H})$, $3.27\left(\mathrm{~d}, J_{\mathrm{P} . \mathrm{H}}=1,2 \mathrm{H}\right), 2.69(\mathrm{dd}, J=17,11,2 \mathrm{H}), 2.60(\mathrm{dd}, J=$ $15,3 \mathrm{~Hz}, 2 \mathrm{H}), 1.40(\mathrm{br}, 1 \mathrm{H})$ and $0.71(\mathrm{~s}, 3 \mathrm{H}):{ }^{31} \mathrm{P}, \delta 40.3$.

[2,2-Bis(diphenylphosphinomethyl)propan-1-ol- $\left.P, P^{\prime}\right]$ tetracarbonylmolybdenum $(0) 2$. White solid $(96 \%)$ (Found: C, 59.55; $\mathrm{H}, 4.65$. Calc. for $\mathrm{C}_{33} \mathrm{H}_{30} \mathrm{MoO}_{5} \mathrm{P}_{2}: \mathrm{C}, 59.65 ; \mathrm{H}, 4.55 \%$ ), m.p. $155-158^{\circ} \mathrm{C}$. IR (benzene): $v_{\mathrm{CO}} 2017,1957,1896$ and $1809 \mathrm{~cm}^{-1}$. NMR: ${ }^{1} \mathrm{H}, \delta 7.87-7.80(\mathrm{~m}, 4 \mathrm{H}), 7.52-7.32(\mathrm{~m}, 16 \mathrm{H}), 3.35$ $(\mathrm{s}, 2 \mathrm{H}), 2.86(\mathrm{dd}, J=15,12,2 \mathrm{H}), 2.57(\mathrm{dd}, J=15,3.8 \mathrm{~Hz}$, $2 \mathrm{H}), 1.40(\mathrm{br}, 1 \mathrm{H})$ and $0.71(\mathrm{~s}, 3 \mathrm{H}) ;{ }^{31} \mathrm{P}, \delta 18.9$.

$\left[2,2\right.$-Bis(diphenylphosphinomethyl)propan-1-ol- $\left.P, P^{\prime}\right]$ tetracarbonyltungsten(0) 3. Light yellow solid (97\%) (Found: C, 52.55; $\mathrm{H}, 4.00$. Calc. for $\mathrm{C}_{33} \mathrm{H}_{30} \mathrm{O}_{5} \mathrm{P}_{2} \mathrm{~W}: \mathrm{C}, 52.65 ; \mathrm{H}, 4.00 \%$ ), m.p. $168-173{ }^{\circ} \mathrm{C}$. IR (benzene): $v_{\text {CO }} 2013,1948,1916$ and 1886 $\mathrm{cm}^{-1}$. NMR: ${ }^{1} \mathrm{H}, \delta 7.85-7.79(\mathrm{~m}, 4 \mathrm{H}), 7.52-7.34(\mathrm{~m}, 16 \mathrm{H}), 3.34$ (s, $2 \mathrm{H}), 2.94$ (dd, $J=17,15,2 \mathrm{H}), 2.66(\mathrm{dd}, J=15,5 \mathrm{~Hz}, 2 \mathrm{H})$, $1.39(\mathrm{br}, 1 \mathrm{H})$ and $0.73(\mathrm{~s}, 3 \mathrm{H}) ;{ }^{31} \mathrm{P}, \delta-0.4\left(J_{\mathrm{P} \mathrm{w}}=224 \mathrm{~Hz}\right)$.

anti,fac- and syn,fac-[2,2-Bis(diphenylphosphinomethyl)propan-1-ol-P, $\mathrm{P}^{\prime}$ ]bromotricarbonylmanganese(I) $4 \mathbf{a}$ and $\mathbf{4 b}$. A mixture of $\left[\mathrm{Mn}(\mathrm{CO})_{5} \mathrm{Br}\right](527.9 \mathrm{mg}, 1.92 \mathrm{mmol})$ and $\mathrm{dpmp}$ $(875.6 \mathrm{mg}, 1.19 \mathrm{mmol})$ in anhydrous thf $\left(60 \mathrm{~cm}^{3}\right)$ was heated to reflux for $2 \mathrm{~h}$. The reaction mixture was concentrated and the residue filtered through silica gel $(20 \mathrm{~g})$ with acetone as the eluent. The filtrate was concentrated to give an orange-yellow solid $(1281 \mathrm{mg}, 99 \%)$, which was a mixture of $\mathbf{4 a}$ and $\mathbf{4 b}$. Recrystallization from thf-hexane gave $\mathbf{4 b}$ as an orange solid $\left(620 \mathrm{mg}\right.$ ) (Found: $\mathrm{C}, 57.15 ; \mathrm{H}, 4.40$. Calc. for $\mathrm{C}_{32} \mathrm{H}_{30} \mathrm{BrMn}-$ $\mathrm{O}_{4} \mathrm{P}_{2}$ : C, $56.90 ; \mathrm{H}, 4.50 \%$ ), m.p. $183-186{ }^{\circ} \mathrm{C}$ (decomp.). IR $\left(\mathrm{CDCl}_{3}\right): v_{\mathrm{CO}} 2029 \mathrm{~s}, 1963 \mathrm{~m}$ and $1904 \mathrm{~m} \mathrm{~cm}{ }^{1}$. NMR: ${ }^{1} \mathrm{H}\left[\left(\mathrm{CD}_{3}\right)_{2} \mathrm{CO}\right], \delta 7.88-7.82(\mathrm{~m}, 4 \mathrm{H}), 7.61-7.45(\mathrm{~m}, 16 \mathrm{H}), 4.23$ $(\mathrm{br}, 1 \mathrm{H}), 3.30(\mathrm{~s}, 2 \mathrm{H}), 2.74\left(\mathrm{dd}, J=14, J_{\mathrm{P}-\mathrm{C}-\mathrm{H}}=3.3,2 \mathrm{H}\right), 2.59$ (dd, $\left.J=14, J_{\mathrm{P}-\mathrm{C}-\mathrm{H}}=8.7 \mathrm{~Hz}, 2 \mathrm{H}\right)$ and $0.26(\mathrm{~s}, 3 \mathrm{H}) ;{ }^{11} \mathrm{P}, \delta 29.7$. 4a. IR $\left(\mathrm{CDCl}_{3}\right): v_{\mathrm{CO}} 2029 \mathrm{~s}, 1963 \mathrm{~m}$ and $1904 \mathrm{~m} \mathrm{~cm}^{-1}$. NMR: ${ }^{1} \mathrm{H}\left[\left(\mathrm{CD}_{3}\right)_{2} \mathrm{CO}\right], \delta 7.80-7.78(\mathrm{~m}, 4 \mathrm{H}), 7.69-7.65(\mathrm{~m}, 4 \mathrm{H})$, $7.54-7.37(\mathrm{~m}, 12 \mathrm{H}), 3.96(\mathrm{br}, 1 \mathrm{H}), 3.06\left(\mathrm{dd}, J=14, J_{\mathrm{P} \mathrm{C} \mathrm{н}}=\right.$ $4.2,2 \mathrm{H}), 2.86(\mathrm{~s}, 2 \mathrm{H}), 2.68\left(\mathrm{dd}, J=14, J_{\mathrm{P}-\mathrm{C}-\mathrm{H}}=6.5 \mathrm{~Hz}, 2 \mathrm{H}\right)$ and $1.01(\mathrm{~s}, 3 \mathrm{H}) ;{ }^{31} \mathrm{P}, \delta 29.7$.

Table 6 Torsional angles $\left({ }^{\circ}\right)$ along the chelate ring

Complex 4b
$\mathrm{Mn}-\mathrm{P}(1)-\mathrm{C}(1)-\mathrm{C}(2)-\mathrm{C}(3)-\mathrm{P}(2)$

$\begin{array}{lr}\mathrm{Mn}-\mathrm{P}(1)-\mathrm{C}(1)-\mathrm{C}(2) & -58.8(3) \\ \mathrm{P}(1)-\mathrm{C}(1)-\mathrm{C}(2)-\mathrm{C}(3) & 60.4(4) \\ \mathrm{C}(1)-\mathrm{C}(2)-\mathrm{C}(3)-\mathrm{P}(2) & -61.2(4) \\ \mathrm{C}(2)-\mathrm{C}(3)-\mathrm{P}(2)-\mathrm{Mn} & 61.2(3) \\ \mathrm{C}(3)-\mathrm{P}(2)-\mathrm{Mn}-\mathrm{P}(1) & -43.7(2) \\ \mathrm{P}(2)-\mathrm{Mn}-\mathrm{P}(1)-\mathrm{C}(1) & 42.4(2)\end{array}$

Complex 9b

$\mathrm{Mn}-\mathrm{P}(1)-\mathrm{C}(1)-\mathrm{C}(2)-\mathrm{C}(3)-\mathrm{P}(2)$

$\begin{array}{lr}\mathrm{Mn}-\mathrm{P}(1)-\mathrm{C}(1)-\mathrm{C}(2) & -51.5(4) \\ \mathrm{P}(1)-\mathrm{C}(1)-\mathrm{C}(2)-\mathrm{C}(3) & 50.9(5) \\ \mathrm{C}(1)-\mathrm{C}(2)-\mathrm{C}(3)-\mathrm{P}(2) & -56.5(5) \\ \mathrm{C}(2)-\mathrm{C}(3)-\mathrm{P}(2)-\mathrm{Mn} & 63.8(4) \\ \mathrm{C}(3)-\mathrm{P}(2)-\mathrm{Mn}-\mathrm{P}(1) & -48.1(3) \\ \mathrm{P}(2)-\mathrm{Mn}-\mathrm{P}(1)-\mathrm{C}(1) & 42.5(3)\end{array}$

Complex 6

$\mathrm{Mn}-\mathrm{P}(1)-\mathrm{C}(1)-\mathrm{C}(2)-\mathrm{C}(3)-\mathrm{P}(2)$

$\begin{array}{lr}\mathrm{Mn}-\mathrm{P}(1)-\mathrm{C}(1)-\mathrm{C}(2) & -34.0(2) \\ \mathrm{P}(1)-\mathrm{C}(1)-\mathrm{C}(2)-\mathrm{C}(3) & -43.5(2) \\ \mathrm{C}(1)-\mathrm{C}(2)-\mathrm{C}(3)-\mathrm{P}(2) & 80.5(3) \\ \mathrm{C}(2)-\mathrm{C}(3)-\mathrm{P}(2)-\mathrm{Mn} & -24.9(2) \\ \mathrm{C}(3)-\mathrm{P}(2)-\mathrm{Mn}-\mathrm{P}(1) & -36.1(1) \\ \mathrm{P}(2)-\mathrm{Mn}-\mathrm{P}(1)-\mathrm{C}(1) & 63.8(1)\end{array}$

$\mathrm{Mn}-\mathrm{P}(1)-\mathrm{C}(1)-\mathrm{C}(2)-\mathrm{C}(5)-\mathrm{O}(5)$

$\begin{array}{lr}\mathrm{Mn}-\mathrm{P}(1)-\mathrm{C}(1)-\mathrm{C}(2) & -34.0(2) \\ \mathrm{P}(1)-\mathrm{C}(1)-\mathrm{C}(2)-\mathrm{C}(5) & 81.6(3) \\ \mathrm{C}(1)-\mathrm{C}(2)-\mathrm{C}(5)-\mathrm{O}(5) & -49.7(3) \\ \mathrm{C}(2)-\mathrm{C}(5)-\mathrm{O}(5)-\mathrm{Mn} & -28.0(2) \\ \mathrm{C}(5)-\mathrm{O}(5)-\mathrm{Mn}-\mathrm{P}(1) & 58.2(2) \\ \mathrm{O}(5)-\mathrm{Mn}-\mathrm{P}(1)-\mathrm{C}(1) & -19.2(2)\end{array}$

$\mathrm{Mn}-\mathrm{P}(2)-\mathrm{C}(3)-\mathrm{C}(2)-\mathrm{C}(5)-\mathrm{O}(5)$

$\begin{array}{lr}\mathrm{Mn}-\mathrm{P}(2)-\mathrm{C}(3)-\mathrm{C}(2) & -24.9(2) \\ \mathrm{P}(2)-\mathrm{C}(3)-\mathrm{C}(2)-\mathrm{C}(5) & -42.3(2) \\ \mathrm{C}(3)-\mathrm{C}(2)-\mathrm{C}(5)-\mathrm{O}(5) & 75.5(4) \\ \mathrm{C}(2)-\mathrm{C}(5)-\mathrm{O}(5)-\mathrm{Mn} & -28.0(2) \\ \mathrm{C}(5)-\mathrm{O}(5)-\mathrm{Mn}-\mathrm{P}(2) & -29.4(2) \\ \mathrm{O}(5)-\mathrm{Mn}-\mathrm{P}(2)-\mathrm{C}(3) & 49.3(2)\end{array}$


Table 7 Crystal data for complexes $4 \mathrm{~b}, 6$ and $9 b^{a}$

\begin{tabular}{|c|c|c|c|}
\hline $\begin{array}{l}\text { Compound } \\
\text { Formula } \\
M\end{array}$ & $\begin{array}{l}\text { 4b-thf } \\
\mathrm{C}_{36} \mathrm{H}_{38} \mathrm{BrMnO}_{5} \mathrm{P}_{2} \\
747.47\end{array}$ & $\begin{array}{l}\text { 6. } \mathrm{Me}_{2} \mathrm{CO} \\
\mathrm{C}_{35} \mathrm{H}_{36} \mathrm{~F}_{6} \mathrm{MnO}_{5} \mathrm{P}_{3} \\
798.54\end{array}$ & $\begin{array}{l}9 \mathrm{~b} \\
\mathrm{C}_{33} \mathrm{H}_{30} \mathrm{MnNO}_{4} \mathrm{P}_{2} \mathrm{~S} \\
653.55\end{array}$ \\
\hline Crystal system & Monoclinic & Monoclinic & Triclinic \\
\hline Crystal size $/ \mathrm{mm}$ & $0.40 \times 0.50 \times 0.50$ & $0.35 \times 0.35 \times 0.35$ & $0.15 \times 0.20 \times 0.2$ \\
\hline Space group & $P 2_{1} / c$ & $C c$ & $P \bar{\top}$ \\
\hline a/ $\AA \AA$ & $11.488(2)$ & $21.293(8)$ & $9.981(5)$ \\
\hline$b / \AA$ & 17.171(3) & $9.939(5)$ & $12.228(4)$ \\
\hline$c / \AA ̊$ & $18.209(8)$ & $18.520(9)$ & $14.198(7)$ \\
\hline$\alpha /^{\circ}$ & - & - & $77.25(3)$ \\
\hline$\beta /^{\circ}$ & $102.56(2)$ & $111.62(4)$ & $77.00(4)$ \\
\hline$\gamma /{ }^{\circ}$ & - & - & $68.23(4)$ \\
\hline$U / \AA^{3}$ & $3506(1)$ & $3627(3)$ & $1550(1)$ \\
\hline$Z$ & 4 & 4 & 2 \\
\hline$D_{\mathrm{c}} / \mathrm{g} \mathrm{cm}^{-3}$ & 1.416 & 1.463 & 1.401 \\
\hline$\mu / \mathrm{mm}^{-1}$ & 1.61 & 0.54 & 0.61 \\
\hline $2 \theta$ range ${ }^{\circ}$ & $19.06-22.20$ & $18.7-28.60$ & $19.06-23.04$ \\
\hline$F(000)$ & 1536 & 1640 & 676 \\
\hline$T / \mathrm{K}$ & 298 & 298 & 298 \\
\hline Scan width ${ }^{b}$ & $0.8+0.35 \tan \theta$ & $0.7+0.35 \tan \theta$ & $0.75+0.35 \tan \theta$ \\
\hline Transmission range & $0.92-1.00$ & $0.97-1.00$ & $0.71-1.00$ \\
\hline $2 \theta_{\max } /^{\circ}$ & 45.0 & 50.0 & 45.0 \\
\hline No. of unique reflections & 4597 & 3187 & 3766 \\
\hline No. of reflections observed $[I>2 \sigma(I)]$ & 2785 & 2596 & 2142 \\
\hline No. of parameters & 407 & 454 & 380 \\
\hline$R$ & 0.042 & 0.034 & 0.040 \\
\hline$R^{\prime}$ & 0.039 & 0.024 & 0.034 \\
\hline$S$ & 1.95 & 1.80 & 1.31 \\
\hline
\end{tabular}

${ }^{a}$ Details in common: $\theta-2 \theta$ scan mode, Mo-K $\alpha$ radiation $(\lambda=0.71069 \AA)$; weighting scheme $w^{-1}=\sigma^{2}(F){ }^{b}$ The stationary counting for background is applied.

anti,fac- and syn,fac-[2,2-Bis(diphenylphosphinomethyl)propan-1-ol- $\left.-\mathrm{P}^{\prime}\right]$ tricarbonyliodomanganese( $\left.\mathrm{I}\right) \mathbf{5 a}$ and $\mathbf{5 b}$. Complexes $\mathbf{5 a}$ and $\mathbf{5 b}$ were obtained in a similar procedure to that described for 4a and 4b. 5a. IR $\left(\mathrm{CDCl}_{3}\right): v_{\mathrm{CO}} 2023 \mathrm{~s}, 1961 \mathrm{~m}$ and $1905 \mathrm{~m} \mathrm{~cm}^{-1}$. NMR $\left[\left(\mathrm{CD}_{3}\right)_{2} \mathrm{CO}\right]:{ }^{1} \mathrm{H}, \delta 7.76-7.74(\mathrm{~m}, 4 \mathrm{H})$, 7.72-7.70 (m, $4 \mathrm{H}), 7.49-7.35(\mathrm{~m}, 12 \mathrm{H}), 3.75(\mathrm{br}, 1 \mathrm{H}), 3.25(\mathrm{dd}$, $\left.J=14, J_{\mathrm{P}-\mathrm{C}-\mathrm{H}}=3.3,2 \mathrm{H}\right), 2.84\left(\mathrm{dd}, J=14, J_{\mathrm{P}-\mathrm{C}-\mathrm{H}}=6.5 \mathrm{~Hz}\right.$, $2 \mathrm{H}), 2.78(\mathrm{~s}, 2 \mathrm{H})$ and $1.08(\mathrm{~s}, 3 \mathrm{H}) ;{ }^{31} \mathrm{P}, \delta 26.4$. 5b (Found: $\mathrm{C}$, $52.80 ; \mathrm{H}, 4.65$. Calc. for $\mathrm{C}_{32} \mathrm{H}_{30} \mathrm{IMnO}_{4} \mathrm{P}_{2}: \mathrm{C}, 53.20 ; \mathrm{H}, 4.20 \%$, m.p. $184-189^{\circ} \mathrm{C}$ (decomp.). IR $\left(\mathrm{CDCl}_{3}\right): v_{\mathrm{CO}} 2023 \mathrm{~s}, 1961 \mathrm{~m}$ and $1905 \mathrm{~m} \mathrm{~cm}^{-1}$. NMR $\left[\left(\mathrm{CD}_{3}\right)_{2} \mathrm{CO}\right]:{ }^{1} \mathrm{H}, \delta 7.93-7.88(\mathrm{~m}, 4 \mathrm{H})$, 7.56-7.51 (m, $4 \mathrm{H}), 7.49-7.35(\mathrm{~m}, 12 \mathrm{H}), 4.38$ (br, $1 \mathrm{H}), 3.65$ $\left(\mathrm{dd}, J=14, J_{\mathrm{P}-\mathrm{C}-\mathrm{H}}=3.3,2 \mathrm{H}\right), 3.34(\mathrm{~s}, 2 \mathrm{H}), 2.63(\mathrm{dd}, J=14$, $\left.J_{\mathrm{P}-\mathrm{C}-\mathrm{H}}=8.8 \mathrm{~Hz}, 2 \mathrm{H}\right)$ and $0.16(\mathrm{~s}, 3 \mathrm{H}) ;{ }^{31} \mathrm{P}, \delta 26.4$.

fac-[2,2-Bis(diphenylphosphinomethyl) propan-1-ol-P, $\left.\mathrm{P}^{\prime}, \mathrm{O}\right]-$ tricarbonylmanganese( $\mathrm{I})$ hexafluorophosphate 6 . To a flask containing $\mathrm{AgPF}_{6}(355.5 \mathrm{mg}, 1.41 \mathrm{mmol})$ was added a solution of a mixture of $4 \mathbf{a}$ and $4 \mathbf{b}(948.9 \mathrm{mg}, 1.41 \mathrm{mmol})$ in thf $\left(30 \mathrm{~cm}^{3}\right)$. After stirring for $0.5 \mathrm{~h}$, the reaction mixture was filtered through silica gel $(20 \mathrm{~g})$ and washed with acetone. The filtrate was concentrated and the residue was recrystallized from thfhexane to give the desired complex as a yellow crystalline solid $(1030 \mathrm{mg}, 90 \%)$ (Found: C, 52.00; H, 4.45. Calc. for $\mathrm{C}_{32} \mathrm{H}_{30} \mathrm{~F}_{6} \mathrm{MnO}_{4} \mathrm{P}_{3}: \mathrm{C}, 51.90 ; \mathrm{H}, 4.10 \%$ ), m.p. $163-167^{\circ} \mathrm{C}$ (decomp.). IR $\left(\mathrm{CDCl}_{3}\right): v_{\mathrm{CO}} 2040 \mathrm{~s}, 1973 \mathrm{~m}$ and $1923 \mathrm{~m} \mathrm{~cm}^{-1}$. NMR $\left[\left(\mathrm{CD}_{3}\right) \mathrm{CO}\right]:{ }^{1} \mathrm{H}, \delta$ 7.54-7.51 (m, $\left.16 \mathrm{H}\right), 7.36-7.31(\mathrm{~m}$, $4 \mathrm{H}), 7.07(\mathrm{br}, 1 \mathrm{H}), 3.71(\mathrm{~s}, 2 \mathrm{H}), 3.03\left(\mathrm{dd}, J=16, J_{\mathrm{P}-\mathrm{C}-\mathrm{H}}=5.7\right.$, $2 \mathrm{H}), 2.53\left(\mathrm{dd}, J=16, J_{\mathrm{P}-\mathrm{C}-\mathrm{H}}=4.7 \mathrm{~Hz}, 2 \mathrm{H}\right)$ and $1.34(\mathrm{~s}, 3 \mathrm{H})$; ${ }^{31} \mathrm{P}, \delta 34.8$

anti,fac- and syn,fac-[2,2-Bis(diphenylphosphinomethyl)propyl methyl ether $\left.-\mathrm{P}, \mathrm{P}^{\prime}\right]$ bromotricarbonylmanganese(I) 7a and 7b. These complexes were prepared according to a similar procedure to that described for complexes $4 \mathbf{a}$ and $4 \mathbf{b}$. 7a (Found: C, 57.45; H, 4.70. Calc. for $\mathrm{C}_{33} \mathrm{H}_{32} \mathrm{BrMnO}_{4} \mathrm{P}_{2}: \mathrm{C}, 57.50 ; \mathrm{H}$, $4.70 \%)$. IR $\left(\mathrm{CHCl}_{3}\right): v_{\mathrm{CO}} 2030 \mathrm{~s}, 1966 \mathrm{~m}$ and $1907 \mathrm{~m} \mathrm{~cm}^{-1}$. NMR: ${ }^{1} \mathrm{H}, \delta 7.68-7.64(\mathrm{~m}, 8 \mathrm{H}), 7.42-7.33(\mathrm{~m}, 12 \mathrm{H}), 3.12(\mathrm{~d}, J=14$, $2 \mathrm{H}), 2.57(\mathrm{~s}, 3 \mathrm{H}), 2.51\left(\mathrm{dt}, J=14, J_{\mathrm{P}-\mathrm{C}-\mathrm{H}}=7.1 \mathrm{~Hz}, 2 \mathrm{H}\right), 2.31$ (s, $2 \mathrm{H}$ ) and $1.12(\mathrm{~s}, 3 \mathrm{H}) ;{ }^{31} \mathrm{P}, \delta 30.2$. 7b, m.p. $190-193{ }^{\circ} \mathrm{C}$ (decomp.). IR $\left(\mathrm{CHCl}_{3}\right): v_{\mathrm{CO}} 2030 \mathrm{~s}, 1966 \mathrm{~m}$ and $1907 \mathrm{~m} \mathrm{~cm}^{-1}$.
NMR: ${ }^{1} \mathrm{H}\left(\mathrm{CDCl}_{3}\right), \delta 7.68-7.64(\mathrm{~m}, 8 \mathrm{H}), 7.42-7.33(\mathrm{~m}, 12 \mathrm{H})$, $3.27(\mathrm{~d}, J=14,2 \mathrm{H}), 2.97(\mathrm{~s}, 5 \mathrm{H}), 2.28\left(\mathrm{dt}, J=14, J_{\mathrm{P}-\mathrm{C}-\mathrm{H}}=\right.$ $7.5 \mathrm{~Hz}, 2 \mathrm{H})$ and $0.46(\mathrm{~s}, 3 \mathrm{H}) ;{ }^{31} \mathrm{P}, \delta 29.2$.

Ligand-substitution reactions of complex 6 by halides and pseudo-halides. To a solution of complex 6 in anhydrous thf or acetone was added $\mathrm{NBu}_{4} \mathrm{X}(\mathrm{X}=\mathrm{Br}$ or $\mathrm{SCN}), \mathrm{KI}$ or $\mathrm{NaN}_{3}$ at room temperature. After stirring for $30 \mathrm{~min}$, the solvent was removed and the residue was dissolved in $\mathrm{CDCl}_{3}$ to measure the ${ }^{1} \mathrm{H}$ NMR spectrum. Complexes $\mathbf{4 b}$ and $\mathbf{5 b}$ were identified by spectral methods, whereas complexes $8 \mathrm{~b}$ and $\mathbf{9 b}$ were isolated by recrystallization from thf-hexane.

syn, fac-Azido[2,2-bis(diphenylphosphinomethyl)propan-1ol- $\left.P, P^{\prime}\right]$ tricarbonylmanganese(I) $\mathbf{8 b}$. Yellow solid (Found: C, 59.90; $\mathrm{H}, 4.90 ; \mathrm{N}, 6.30$. Calc. for $\mathrm{C}_{32} \mathrm{H}_{30} \mathrm{MnN}_{3} \mathrm{O}_{4} \mathrm{P}_{2}$ : C, $60.30 ; \mathrm{H}, 4.75 ; \mathrm{N}, 6.60 \%)$, m.p. $152-155^{\circ} \mathrm{C}$. IR: $v(\mathrm{~N}=\mathrm{N}) 2053$; $v(C O) 2009 \mathrm{~s}, 1999 \mathrm{~m}, 1948 \mathrm{~m}$ and $1903 \mathrm{~m} \mathrm{~cm}^{-1}$. NMR: ${ }^{1} \mathrm{H}\left[\left(\mathrm{CD}_{3}\right) \mathrm{CO}\right], \delta$ 7.86-7.80 (m, $\left.4 \mathrm{H}\right), 7.55-7.43(\mathrm{~m}, 16 \mathrm{H}), 4.20$ (br, $1 \mathrm{H}$ ), 3.23 (s, $2 \mathrm{H}$ ), 2.92 (dd, $J=14.6,3.5,2 \mathrm{H}$ ), 2.41 (dd, $J=14.6,7.9 \mathrm{~Hz}, 2 \mathrm{H})$ and $0.42(\mathrm{~s}, 3 \mathrm{H}) ;{ }^{31} \mathrm{P}, \delta 32.7$.

syn,fac-[2,2-Bis(diphenylphosphinomethyl)propan-1-ol$\left.P, P^{\prime}\right]$ tricarbonyl(thiocyanato- $N$ ) manganese(I) $9 \mathrm{~b}$. Light yellow solid (Found: $\mathrm{C}, 60.50 ; \mathrm{H}, 4.75 ; \mathrm{N}, 2.10$. Calc. for $\mathrm{C}_{33^{-}}$ $\mathrm{H}_{30} \mathrm{MnNO}_{4} \mathrm{P} 2 \mathrm{~S}: \mathrm{C}, 60.65 ; \mathrm{H}, 4.65 ; \mathrm{N}, 2.15^{\circ}$ ), m.p. $186-189^{\circ} \mathrm{C}$ (decomp.). IR:v(NCS) 2105, v(CO) 2036s, 1968m and $1926 \mathrm{~m}$ $\mathrm{cm}^{-1}$. NMR: ${ }^{1} \mathrm{H}, \delta$ 7.88-7.82(m, $\left.4 \mathrm{H}\right), 7.61-7.45(\mathrm{~m}, 16 \mathrm{H}), 4.23$ (br, $1 \mathrm{H}), 3.35(\mathrm{~s}, 2 \mathrm{H}), 2.74$ (dd, $J=14,3.3,2 \mathrm{H}), 2.59$ (dd, $J=$ $14,8.7 \mathrm{~Hz}, 2 \mathrm{H})$ and $0.45(\mathrm{~s}, 3 \mathrm{H}) ;{ }^{31} \mathrm{P}, \delta 33.4$.

$X$-Ray Crystallography.-Crystals of $4 \mathrm{~b}, 6$ and 9b were obtained in suitable forms for X-ray analysis. Cell parameters were measured and data collected on a CAD4 diffractometer. Scattering factors were taken from ref. 23 and the NRCC SDP VAX program was used for the calculations. ${ }^{24}$ Details of the crystal parameters, data collection and structure refinement for complexes $4 \mathrm{~b}, 6$ and $9 \mathrm{~b}$ are summarized in Table 7. Complex 6 crystallized in a monoclinic space group with a $C$ centre and $c$ glide; the intensity distribution favoured the non-centric one. The space group $C c$ was assumed and confirmed by the successful solution and refinement of the structure. 
Additional material available from the Cambridge Crystallographic Data Centre comprises thermal parameters and remaining bond lengths and angles.

\section{Acknowledgements}

We thank the National Science Council of the Republic of China (NSC83-0208-M-002-63) for financial support.

\section{References}

1 A. Bader and E. Lindner, Coord. Chem. Rev., 1991, 108, 27.

2 M. R. Mason, Y. Su, R. A. Jacobson and J. G. Verkade, Organometallics, 1991, 10, 2335; M. R. Mason and J. G. Verkade, Organometallics, 1992, 11, 1514.

3 V.V. S. Reddy, A. Varshney and G. M. Gray, J. Organomet. Chem., 1990, 391, 259; A. Varshney, M. L. Webster and G. M. Gray, Inorg. Chem. 1992, 31, 2580.

4 E. Lindner and R. Speidel, Z. Naturforsch., Teil B, 1989, 44, 437; E. Lindner, H. Rothfuß, R. Fawzi and W. Hiller, Chem. Ber., 1992, 125,541 .

5 J. C. Jeffrey and T. B. Rauchfuss, Inorg. Chem., 1979, 18, 2658.

6 G. K. Anderson and R. Kumar, Inorg. Chem., 1984, 23, 4064.

7 S. J. Chen and K. R. Dunbar, Inorg. Chem., 1990, 29, 588.

8 N. W. Alcock, J. M. Brown and J. C. Jeffrey, J. Chem. Soc., Dalton Trans., 1987, 2273.

9 A. J. McAlees, R. McCrindle and A. R. Woon-Fat, Inorg. Chem., $1976,15,1065$.
10 H. E. Bryndza and W. Tam, Chem. Rev., 1988, 88, 1163.

11 D. Harry, M. W. Thewissen, K. Timmer, J. G. Noltes and J. W. Marsman, Inorg. Chim. Acta, 1985, 97, 143.

12 U. Klabunde and S. D. Ittel, J. Mol. Catal., 1987, 41, 123

$13 \mathrm{~J}$. Ipaktschi and W. Sulzbach, J. Organomet. Chem., 1992, 434, 287.

14 S.-T. Liu, G.-J. Liu, C.-H. Yieh, M.-C. Cheng and S.-M. Peng, J. Organomet. Chem., 1990, 387, 83.

15 S.-T. Liu, C.-H. Yieh and H.-J. Lu, Phosphorus Sulfur, 1989, 44, 261. 16 S.-T. Liu, H.-E. Wang, L.-M. Yiin, S.-C. Tsai, K.-J. Liu, Y.-M. Wang, C.-M. Cheng and S.-M. Peng, Organometallics, 1993, 12, 2277.

17 C. K. Johnson, ORTEP, Report ORNL-5138, Oak Ridge National Laboratory, Oak Ridge, TN, 1976.

18 S.-C. Tsai, H.-E. Wang, C.-T. Huang, L.-M. Yiin, C.-M. Cheng, S.-M.Peng and S.-T. Liu, Chem. Ber., in the press.

19 H.-E. Wang, M.-C. Cheng, G.-H. Lee, S.-M. Peng and S.-T. Liu, J. Organomet. Chem., 1993, 445, 171.

20 B. Beagley, C. A. McAuliffe, A. G. Mackie and R. G. Pritchard, Inorg Chim. Acta, 1984, 89, 163.

21 M. J. Coyer, R. H. Herber, J. Chen, M. Croft and S. P. Szu, Inorg. Chem., 1994, 33, 716.

22 J. L. Burmeister, Coord. Chem. Rev., 1990, 105, 77.

23 International Tables for X-Ray Crystallography, Kynoch Press, Birmingham, 1974, vol. 4.

24 E. J. Gabe and F. L. Lee, Acta Crystallogr., Sect. A, 1981, 37, S339.

Received 27th April 1994; Paper 4/02495H 